(2) Open Access Full Text Article

\title{
Poor tolerance of beta-blockers by elderly patients with heart failure
}

This article was published in the following Dove Press journal:

Clinical Interventions in Aging

25 November 2010

Number of times this article has been viewed

\author{
Satoshi Yanagisawa \\ Noriyuki Suzuki \\ Toshikazu Tanaka \\ Department of Cardiology, Okazaki \\ City Hospital, Aichi, Japan
}

\begin{abstract}
Despite the well-understood importance of beta-blocker therapy in heart failure, it is sometimes not possible to use beta-blockers in elderly patients due to poor tolerance. In this report, we describe the case of an 83-year-old patient with severe systolic heart failure complicated by aortic valve stenosis and atrial fibrillation. A simple therapeutic approach involving discontinuation of beta-blockers remarkably alleviated the symptoms such as left ventricular ejection fraction, and improved the chest radiography and laboratory findings; further, atrial fibrillation converted to sinus rhythm. It is important to carefully administer beta-blocker therapy to elderly patients with heart failure, especially after considering cardiac output.

Keywords: elderly, octogenarians, beta-blockers, heart failure
\end{abstract}

\section{Introduction}

It is well established that beta-blocker therapy reduces morbidity and mortality in heart failure patients. ${ }^{1,2}$ With advances in the management of heart disease and the widespread recognition of their effects, beta-blockers are the mainstay of the current treatment for heart failure. However, they can cause adverse effects such as hypotension, bradycardia, and reversible pulmonary disease. Because some patients have poor tolerance towards beta-blockers, dosage reduction or discontinuation is sometimes necessary, despite the advantages of beta-blockers. In this report, we describe the case of a patient with severe heart failure complicated by aortic valve stenosis and atrial fibrillation, which were dramatically improved after discontinuation of beta-blockers.

\section{Case report}

An 83-year-old woman with a history of heart failure and repeated hospitalization due to decompensated heart failure (New York Heart Association [NYHA] functional classification class III) was transferred to our institution on the recommendation of a nearby general practitioner one year ago. The patient had acute heart failure, and severe complications were recorded after conducting several intensive examinations. The electrocardiogram (ECG) obtained at admission revealed atrial fibrillation with a QS pattern in the V1-V3 leads, indicating an old myocardial infarction. Her mean heart rate was $60-70$ beats/min. Two-dimensional transthoracic echocardiography revealed a slightly dilated left atrium and ventricle, and severe hypokinesis in the left ventricle from the septal to the apical area. The left ventricular ejection fraction (LVEF) was $25 \%$. A severe degree of aortic valve stenosis with calcification was observed (peak velocity, $2.9 \mathrm{~m} / \mathrm{s}$; peak transvalvular gradient, $33 \mathrm{mmHg}$; and indexed
Correspondence: Satoshi Yanagisawa Department of Cardiology, Okazaki City Hospital, 3-I Goshoai Koryuji-cho, Okazaki, Aichi, 444-8553, Japan

Tel +8 I 05642 I 8 III

Fax +81 0564252913

Email pinponstar@yahoo.co.jp 
valve area, $0.55 \mathrm{~cm}^{2}$, indicating reduced blood flow and low-gradient aortic stenosis). Further, a severe degree of tricuspid regurgitation was observed. On the basis of the ECG and echocardiographic findings, we elected to perform catheter angiography that revealed severe coronary artery stenosis in the left anterior descending artery and severe peripheral artery disease (total occlusion of the left iliac artery and severe stenosis in the superficial femoral artery). Despite the advanced age of the patient and the presence of comorbidities, we recommended invasive treatment with percutaneous coronary intervention or aortic valve replacement on the basis of catheterization and echocardiographic findings; however, the patient and her family refused the invasive treatment, and she was administered optimal medical therapy. The patient was already taking aspirin, angiotensin-receptor blockers, statin, diuretic agents, and beta-blocker bisoprolol (2.5 mg/day) for coronary artery disease, heart failure, and atrial fibrillation. These medications were continued after discharge.

After discharge from the first hospitalization, her condition gradually worsened. She was hospitalized many times due to heart failure, and diuretic agent dose was gradually increased. The dose of $N$-terminal pro-brain natriuretic peptide (NT-proBNP) was markedly elevated to $8739 \mathrm{pg} / \mathrm{mL}$, suggesting severe heart failure that was difficult to control. A chest radiograph showed cardiomegaly with a cardiothoracic ratio of $71 \%$ (Figure $1 \mathrm{~A}$ ).

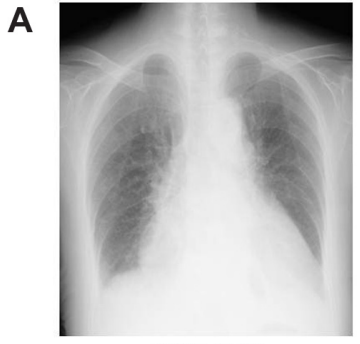

(CTR, 71\%)

Before bisoprolol discontinuation

B

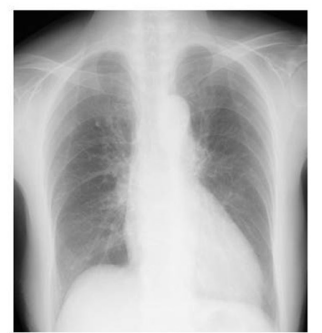

(CTR, 57\%)

After bisoprolol discontinuation

Figure I At the time of the most severe condition, the chest radiograph showed cardiomegaly with cardiothoracic ratio (CTR) of $71 \%$; an electrocardiogram revealed atrial fibrillation with a QS pattern in the VI-V3 leads $\mathbf{A}$ ). After bisoprolol discontinuation, the CTR determined by chest radiography was reduced to $57 \%$, and atrial fibrillation converted to sinus rhythm B).
She was confined to a wheelchair with care support and could not walk by herself because of dyspnea, chest pain, and lack of physical activity. We carefully re-evaluated her condition, and observed a decreased heart rate of less than 60 beats $/ \mathrm{min}$. Bisoprolol was discontinued on suspicion that it caused bradycardia, which indicated very poor heart function.

After bisoprolol discontinuation, the condition of the patient gradually improved. Dyspnea and effort angina disappeared, and she could walk by herself without care support. Her functional capacity improved to NYHA class II. Two months after bisoprolol discontinuation, transthoracic echocardiography revealed a significant improvement of $62 \%$ in the LVEF and a marked reduction in the tricuspid regurgitation degree. The cardiothoracic ratio, determined by chest radiography, was reduced to $57 \%$ (Figure 1B). The NT-proBNP level was 2962 pg/mL. Finally, atrial fibrillation was spontaneously terminated, and sinus rhythm was maintained; the mean heart rate was approximately $50-60$ beats $/ \mathrm{min}$. The total clinical course and treatment of the patient are summarized in Figure 2.

\section{Discussion}

We report a case of a patient with heart failure who showed remarkable improvement following discontinuation of betablocker therapy. Not only did heart failure symptoms and quality of life improve but also objective results such as ECG, chest radiography, laboratory test, and echocardiography findings clearly improved.

There is a great deal of evidence of the clinical efficacy of beta-blockers in the young general population; however, few studies have investigated its efficacy in elderly patients
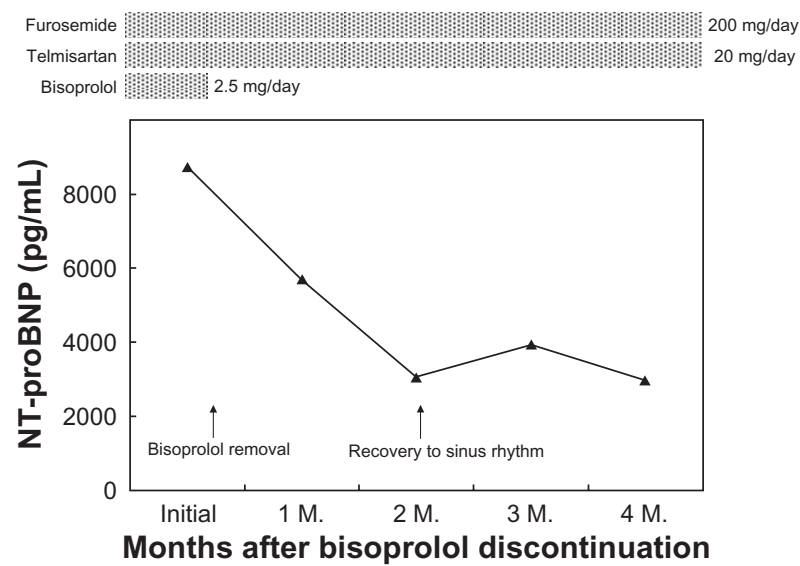

Figure 2 Clinical course and treatment of the patient, and the change in $\mathrm{N}$-terminal pro-brain natriuretic peptide (NT-proBNP). After bisoprolol discontinuation, NT-proBNP gradually decreased. Approximately 2 months later, atrial fibrillation had spontaneously converted to sinus rhythm. 
with heart failure. The SENIORS trial is the only trial that has evaluated the effects of the beta-blocker nebivolol in elderly heart failure patients aged more than 70 years (mean age, 76 years). ${ }^{3}$ The study showed that nebivolol significantly reduced the risk of death or hospitalization. However, the overall risk reduction for the primary endpoint appeared to be less effective than that reported by other studies on younger patients. Another study showed that in patients with advanced chronic heart failure the relative risk reduction of all-cause death was significantly lower in older patients than in younger patients. ${ }^{4}$ In their study (mean age, 76 years), the risk reduction by use of beta-blockers decreases with age from $71 \%$ in patients aged $\leqq 75$ years to $21 \%$ in patients aged $>75$ years. Although the effects of beta-blockers on mortality may be smaller in older patients than younger patients, these agents are still effective in improving mortality and hospitalization rates in elderly patients with heart failure.

In addition, sub-optimal doses of beta-blockers are often used in elderly patients with chronic heart failure. Numerous studies have shown that evidence-based heart failure therapies are underused, especially in the elderly patients. ${ }^{5,6}$ However, there are no large randomized trials that studied the relationship between dose-response and mortality in patients with heart failure. MOCHA, a relatively small study, was designed to evaluate dose-related effects of carvedilol in patients with moderate heart failure and found a dose-related improvement in mortality and LVEF. ${ }^{7}$ On the other hand, subgroup analyses from large trials did not show dose-relative effects on survival, but even low doses conferred benefit in their studies compared to placebo. ${ }^{8,9}$ Thus, even if low doses of beta-blocker can be tolerated by elderly patients, their prescription is encouraged, and therapy is continued.

Our patient was receiving beta-blockers because of complication of coronary heart disease and heart failure due to low LVEF, which was expected to prevent severe outcomes. However, bradycardia, an adverse effect of beta-blockers, was considered to worsen the condition of heart failure. Bradycardia is one of the main causes of beta-broker treatment withdrawal. Even in patients without manifesting sinus or AV nodal conduction disease, betablockers may result in adverse bradyarrhythmia events. In the MIAMI trial, although patients were pre-excluded if their heart rates were less than 65 beats/min, $12.9 \%$ of patients treated with metoprolol experienced adverse reactions due to bradycardia development. ${ }^{10}$ Because conduction system disease incidence is expected to be higher in older patients and most trials exclude very elderly patients, the prevalence of bradyarrythmia due to beta-blockers may be higher than expected in these patients. ${ }^{11}$

In the present case, although bisoprolol was administered in very small doses and the change in heart rate was relatively small (approximately 10 beats/min), the benefit of bisoprolol discontinuation was beyond our expectation. We speculate that beta-blockers not only decreased heart rate but also excessively restricted left ventricular contraction and markedly decreased cardiac output. Aging is associated with a reduction in the intrinsic heart rate and cardiac output but are generally maintained by increasing stroke volume. ${ }^{12}$ Because heart rate response to exercise effort is reduced in elderly patients, stroke volume plays an important role in conducting cardiac output. In the present case, severe aortic valve stenosis further restricted cardiac output, resulting in lowered systolic cardiac output and decreased LVEF. Bisoprolol discontinuation may contribute to softening of the restricted left ventricular contraction and improvement of the decreased cardiac output. The reason for the restoration of the sinus rhythm was that improved cardiac output decreased volume overload and reduced burden on the heart to terminate atrial fibrillation that had persisted for a long period.

Our approach of treatment for this patient was only the discontinuation of beta-blockers. Although this approach is very simple, it is very important that beta-blockers are administered to elderly patients with caution. The reduced benefit of beta-blockers in elderly patients compared to younger patients can be explained by several factors such as age-induced decrease of response to beta-blockers, high prevalence of comorbidities including atrial fibrillation, and high risk of multiple-cause death. ${ }^{4,12}$ Moreover, slow metabolic processes and declined multiple organ systems could unexpectedly affect drug action response and the incidence of adverse reaction in the elderly. Additionally, some patients may not respond to beta-blockers or these drugs may induce adverse effects, especially in patients with systolic heart failure, depending on cardiac output, as was noted in the present case. Further clinical trials are necessary to assess the appropriate and sufficient conditions in which elderly patients can benefit from receiving beta-blocker therapy, and to establish protocols for betablocker removal.

\section{Disclosure}

No conflicts of interest were declared in relation to this paper. 


\section{References}

1. Packer M, Bristow MR, Cohn JN, et al. US Carvedilol Heart Failure Study Group: The effect of carvedilol on morbidity and mortality in patients with chronic heart failure. N Engl J Med. 1996;334(21): 1349-1355.

2. CIBIS-II Investigators and Committees. The Cardiac Insufficiency Bisoprolol Study II (CIBIS-II): A randomised trial. Lancet. 1999;353(9146):9-13.

3. Flather MD, Shibata MC, Coats AJ, et al. Randomized trial to determine the effect of nebivolol on mortality and cardiovascular hospital admission in elderly patients with heart failure (SENIORS). Eur Heart J. 2005; 26:215-225.

4. Dobre D, DeJongste MJ, Lucas C, et al. Effectiveness of beta-blocker therapy in daily practice patients with advanced chronic heart failure; is there an effect-modification by age? Br J Clin Pharmacol. 2007; 63(3):356-364.

5. Cleland JG, Coletta AP, Torabi A, Clark AL. Clinical trials update from the European Society of Cardiology heart failure meeting 2009: CHANCE, B-Convinced, CHAT, CIBIS-ELD, and Signal-HF. Eur $J$ Heart Fail. 2009;11(8):802-805.

6. Lee DS, Tu JV, Juurlink DN, et al. Risk-treatment mismatch in the pharmacotherapy of heart failure. JAMA. 2005;294(10): $1240-1247$.
7. Bristow MR, Gilbert EM, Abraham WT, et al. Carvedilol produces dose-related improvements in left ventricular function and survival in subjects with chronic heart failure. MOCHA Investigators. Circulation. 1996;94(11):2807-2816.

8. Simon T, Mary-Krause M, Funck-Brentano C, Lechat P, Jaillon P. Bisoprolol dose-response relationship in patients with congestive heart failure: a subgroup analysis in the cardiac insufficiency bisoprolol study (CIBIS II). Eur Heart J. 2003;24(6):552-559.

9. Wikstrand J, Hjalmarson A, Waagstein F, et al; MERIT-HF Study Group. Dose of metoprolol CR/XL and clinical outcomes in patients with heart failure: analysis of the experience in metoprolol $\mathrm{CR} / \mathrm{XL}$ randomized intervention trial in chronic heart failure (MERIT-HF). $J$ Am Coll Cardiol. 2002;40(3):491-498.

10. The MIAMI Trial Research Group. Metoprolol in acute myocardial infarction (MIAMI). A randomised placebo-controlled international trial. Eur Heart J. 1985;6(3):199-226.

11. Goldberger JJ, Bonow RO, Cuffe M, et al. Post-myocardial infarction beta-blocker therapy: the bradycardia conundrum. Rationale and design for the pacemaker and beta-blocker therapy post-MI (PACE-MI) trial. Am Heart J. 2008;155(3):455-464.

12. Mangoni AA, Jackson SH. Age-related changes in pharmacokinetics and pharmacodynamics: basic principles and practical applications. Br J Clin Pharmacol. 2004;57(1):6-14.
Clinical Interventions in Aging

\section{Publish your work in this journal}

Clinical Interventions in Aging is an international, peer-reviewed journal focusing on evidence-based reports on the value or lack thereof of treatments intended to prevent or delay the onset of maladaptive correlates of aging in human beings. This journal is indexed on PubMed Central, MedLine, the American Chemical Society's 'Chemical Abstracts Ser-

\section{Dovepress}

vice' (CAS), Scopus and the Elsevier Bibliographic databases. The manuscript management system is completely online and includes a very quick and fair peer-review system, which is all easy to use. Visit http://www.dovepress.com/testimonials.php to read real quotes from published authors. 